\title{
Neuroscientifically-grounded research for improved human-robot interaction
}

\author{
K. Kompatsiari*, J. Pérez-Osorio*, D. De Tommaso, G. Metta, Senior Member, IEEE, A. Wykowska, \\ Member, IEEE
}

\begin{abstract}
The present study highlights the benefits of using well-controlled experimental designs, grounded in experimental psychology research and objective neuroscientific methods, for generating progress in human-robot interaction (HRI) research. More specifically, we aimed at implementing a well-studied paradigm of attentional cueing through gaze (the so-called "joint attention" or "gaze cueing") in an HRI protocol involving the iCub robot. Similarly to documented results in gaze-cueing research, we found faster response times and enhanced eventrelated potentials of the EEG signal for discrimination of cued, relative to uncued, targets. These results are informative for the robotics community by showing that a humanoid robot with mechanistic eyes and human-like characteristics of the face is in fact capable of engaging a human in joint attention to a similar extent as another human would do. More generally, we propose that the methodology of combining neuroscience methods with an HRI protocol, contributes to understanding mechanisms of human social cognition in interactions with robots and to improving robot design, thanks to systematic and wellcontrolled experimentation tapping onto specific cognitive mechanisms of the human, such as joint attention.
\end{abstract}

\section{INTRODUCTION}

The advanced technological capabilities of robotic systems bear a promise of integration of robots in society in the role of companion and/or assistive technology. This, however, calls for intensified research in Human-Robot Interaction (HRI), as integration into society means not only that the robots need to have advanced skills, but also that the humans shall feel comfortable with their future social interaction partners. Therefore, to understand how the human brain reacts to the robot's social presence, and how it processes information conveyed by a robotic agent, it is crucial to employ (neuro-) scientific methods and experimental designs that would bring valid, reproducible and generalizable results to HRI research [1], [2]. Two common present limitations of HRI consist in often relatively small number of test persons, and the lack of experimental protocols where specific mechanisms of the human cognition are targeted systematically with the use of neuroscientific methods, or methods of experimental psychology [2], [3]. Drawing from psychological or social sciences, Bethel et al. [2] proposed guidelines for human studies methods in HRI, using

\footnotetext{
* These authors contributed equally. The project has received funding from the European Research Council (ERC) under the European Union's Horizon 2020 research and innovation programme (grant awarded to AW, titled "InStance: Intentional Stance for Social Attunement". Grant agreement No: 715058).

All authors are with the Istituto Italiano di Tecnologia, Genova, 16145. $\mathrm{K} . \mathrm{K}$ is affiliated with Ludwig-Maximilians-universität (DE). G.M. is a professor of Cognitive Robotics at the University of Plymouth (UK). A.W. is
}

combination of various measures such as self-assessments, interviews, behavioral measures, psychophysiology measures, and task performance metrics. However, most evaluations of robotic systems by human users consist in selfassessments and behavioral methods [2], and they often lack systematicity [3]. In general, even when other measures are included, the studies lack proper adaptation of psychological paradigms, experimental control, or statistical power in the sample.

\section{A. Investigating Joint Attention in HRI}

Here, we focus on one specific experimental paradigm (attentional cueing), which targets a fundamental mechanism of social cognition, namely joint attention (JA). JA occurs when two individuals share their focus on the same object/event, creating a triadic interaction between the self, the other person and the object/event of interest [4]. It constitutes a basis for higher-level mechanisms of human communication [4]-[7]. JA has been extensively studied in cognitive science using gaze-cueing paradigms [8]-[10]. Traditionally, in such paradigms, a face is presented centrally on a computer screen, and then its gaze is shifted towards a location on the screen. Subsequently, a target appears at the gazed-at location (validly-cued target) or at the opposite location (invalidly-cued target). Response times (RTs) for detection or discrimination of validly-cued targets are typically faster than for the invalidly-cued targets, a phenomenon termed as the gaze-cueing effect (GCE). This is explained in terms of attentional orienting: when the gaze of the centrally presented face stimulus shifts towards a location, attentional focus of the observer moves to that location as well, and therefore processing sensory information at that location is facilitated, as compared to a situation when attentional focus needs to be switched to a location that has not been attended (i.e., it has not been cued by the gaze direction). Interestingly, in case of directional cues provided by gaze, orienting of attention appears to be reflexive, as the validity effect occurs even when the gaze is not informative with respect to target location, or is even more likely to cue invalidly [8], [10].

Although the gaze cueing effects have been long investigated in cognitive psychology with the use of stimuli

an adjunct professor in Engineering Psychology, University of Technology, Luleå University of Technology. Corresponding authors: A.W., phone: +39 0208172 242, email: agnieszka.wykowska@iit.it, K.K., email: kyveli.kompatsiari@iit.it. 
on computer screens, implementations of the gaze cueing paradigm in more naturalistic HRI scenarios have been scarce, and results have not been entirely consistent. On the one hand, a study by Admoni et al [11], examining the effect of anthropomorphism on GCE, showed that directional gaze of two different robots did not elicit reflexive GCE. Similarly, Okumura et al. [12] demonstrated that human gaze towards a location elicited anticipatory gaze shifts of 12-year-old infants, while robots gaze did not have the same effect. On the other hand, Wiese, Wykowska et al. [13] showed that a robot face induced a GCE, but to a smaller extent compared to a human face. Furthermore, in a gaze cueing paradigm involving an embodied humanoid agent (the iCub robot [14]), Wykowska et al. [15] showed GCE, independent of whether participants perceived behavior of the robot as human-like or more mechanistic. In the light of these somewhat mixed results, we proceeded to investigate and later implement human-like gaze behavior on the iCub robot, with the use of neuroscience methods. Our measure of success is whether the robot elicits the same behavioral and neural responses of a human interaction partner.

\section{B. Aim of study}

The aim of the present study was to examine whether the behavioural responses (reaction times) and neural correlates (EEG) typically observed on the gaze cueing paradigm could be observed also in an HRI setup. This posed a substantial challenge, given that the experimental paradigm needed to be adapted to a naturalistic interaction with an embodied robot, while EEG, behavioral measures, stimulus presentation and robot behaviors needed to be integrated in one setup, and synchronized with excellent temporal resolution.

In addition, we aimed at examining how reflexive/ automatic is the gaze cueing effect in an interactive HRI setup. That is, whether GCE occurs although the validity of the gaze direction $(50 \%)$ is not informative with respect to subsequent target location, and whether GCE is stable across different manipulations of the gaze direction prior to the gaze cueing procedure. In more detail, we introduced two conditions prior to the shift of gaze to one of the potential target locations. In one condition, the robot gazed straight ahead (similarly to standard design in experimental psychology), and in another, it looked down (cf. Table I). We were interested in whether GCE would be observed not only in a typical scenario of the agent gazing straight ahead, but also in a slightly different condition, namely when the robot looks down prior to gazing to one of the sides.

\section{Motivation}

Replicating the GCE and its neural correlates in a realistic HRI paradigm constitutes a good example of linking cognitive neuroscience with robotics [16]. This approach is grounded in cognitive neuroscience due to implementation of a classical gaze cueing paradigm and the use of neuroscience methods, while its anchoring in robotics occurs through the use of the humanoid robot iCub in an HRI setting. The results are of significant contribution to both fields of research. In social/cognitive neuroscience, this approach allows for examining the mechanisms of social cognition in ecologically valid, yet well-controlled experimental protocol. Humanoid robots, being embodied agents, increase the naturalness of interaction due to their social presence and sharing joint environment (for reviews on the use of embodied robot to studying human cognition see [3], [16], [17]). At the same time, using a humanoid robot rather than another human interaction partner allows for excellent experimental control, as the robot's behavior can be modified in a controlled and modular way, allowing for a systematic investigation of the impact of subtle behavioral cues on social cognitive mechanisms of humans [18]. Furthermore, a robot can reproduce the exact same behavior over many trials of an experiment - a task impossible for a human.

In terms of contribution to robotics, such approach allows for targeting very specific and well-isolated components of human cognition that are at service during interaction with humanoid robots. This should enable progress in designing robots that are well tuned to the workings of the human brain. Only through understanding such well-defined and specific processes of the human brain, will we be able to target them with an adequate design of robot behavior and appearance. To give an example, if our aim is to design robots that are to assist in therapy for children with autism spectrum disorder (ASD), the best strategy is to understand what specific cognitive mechanisms we are aiming to address (responding to or initiating JA, spatial perspective taking, mentalizing, etc.), and depending on which of them is the focus of the therapy, a robot's behavior (together with training protocol) can be designed to address specifically that mechanism. If the aim of training (for the specific impairment) is predominantly based on JA, the robot should be engaging a child in JA, but not necessarily be additionally too expressive, as this might be overwhelming for a child diagnosed with ASD. In other words, in order to engage in JA, we need to understand the optimal conditions and isolate behavioral parameters of the robot to evoke JA in the user, presumably reducing other characteristics of the robot (in the case of training specifically JA, it could mean reducing e.g., emotional expressiveness), which could evoke other - perhaps interfering - mechanisms of social cognition (e.g., emotional reactions), unless it is demonstrated experimentally that emotional expressions positively influence JA.

In general, thanks to the methods and approach proposed here, it is possible to isolate specific parameters of the robot's design that are best suited for evoking specific mechanisms of human cognition in an HRI scenario.

\section{Experimental Design}

In order to replicate JA and its EEG correlates in HRI, we developed a proof-of-concept study with a variation of gazecueing paradigm using the embodied humanoid iCub with a 3D experimental setting. We focused the design and later analyses on the event-related potentials (ERPs) of the EEG signal, related to the behavioral GCE. ERP "components" (shortly, ERPs) are obtained by averaging over multiple trials EEG activity locked to a given event in the trial sequence (here, we focus on the event of stimulus onset). ERPs provide information about the time dynamics of the brain, as the peaks and troughs of an ERP waveform (ERP components) reflect 
cognitive processing, as it unfolds over time. In particular, we focused on the early sensory $\mathrm{P} 1 / \mathrm{N} 1$ components, locked to the onset of the target [19]. P1 is the first positive-amplitude component around $100 \mathrm{~ms}$ post stimulus onset, while N1 is the first negative-amplitude component peaking around 150$200 \mathrm{~ms}$. Both P1 and N1 are related to sensory processing of the stimulus material, and reflect potential attentional modulations of the sensory processes.

ERP correlates of the GCE have been reported on the early sensory P1/N1 components, locked to the onset of the target [19], and reflect the impact of attentional modulation of the sensory gain mechanism [20], [21], which is thought to increase the signal-to-noise ratio (SNR) for stimuli at attended locations, relative to other locations [22], [23]. In more detail, parieto-occipital P1/N1 components have an earlier onset and increased amplitude for stimuli at cued, relative to uncued locations [24]. While the P1 component reflects a perceptual suppression for ignored locations, the N1 indexes enhanced discriminative processing of stimuli within the focus of attention [21], [25]. Fedota et al. [26] suggest that the N1 effect also reflects top-down modulation of discriminative processing in areas of the ventral visual stream, which is in line with evidence also in more social contexts [27], [28]. In summary, for the purposes of our study, we focus on the $\mathrm{P} 1 / \mathrm{N} 1$ components to understand if the iCub robot is capable of inducing similar attentional mechanisms as another human would do, in a social interaction setup. If the robot were to be perceived akin to a human, then we would expect to find a gaze cue-related modulation of the P1/N1 complex locked to target onset in a (e.g. a letter) discrimination task embedded in an interactive HRI protocol.

\section{METHODS}

\section{A. Participants}

To define the sufficient statistical power of our sample, we conducted an a-priori power analysis [29] for the validity effect, using (i) the effect size $(\mathrm{dz}=0.7)$ calculated from a previous study of a similar setup with $\mathrm{iCub}$ and a gaze cueing procedure [15], (ii) an alfa error equal to .05, and (iii) a power level of .85. This analysis yielded an adequate sample size of 21. In total, 24 healthy right-handed (self-reported handedness) volunteers (mean age $=26.16 \pm 4.02,16$ women) were recruited and reimbursed for their participation. 3 of the initial participants were excluded due to artefacts higher than $30 \%$. All had normal or corrected-to normal vision and provided their informed written consent prior to participation. The data were collected at the Istituto Italiano di Technologia, IIT, Genova. The study was approved by the local ethical committee (Comitato Etico Regione Liguria).

\section{B. Stimuli and Apparatus}

The experiment was performed in an isolated and noiseattenuating room. Participants were seated in front of a desk, $125 \mathrm{~cm}$ away from the robot. iCub's eyes were aligned with participants' eyes at $122 \mathrm{~cm}$ from the floor. Two screens, used for stimulus presentation ( 27 inches), were positioned laterally on the desk (75 cm apart centre-to-centre) at a distance of 105 $\mathrm{cm}$ from the participant's nose apex. The screens were slightly tilted back (by $12^{\circ}$ with reference to the vertical position) and were rotated to the right (right screen) or left (left screen) by $14^{\circ}$ with reference to the lateral position, see Figure 1. The target stimuli were letters $\mathrm{V}$ or $\mathrm{T}\left(3^{\circ} 32^{\prime}\right.$ high, $4^{\circ} 5^{\prime}$ wide, the degrees of stimuli refer to visual angle from the human perspective). iCub was looking at five different locations during the experiment: (1) "rest" - towards a point between the desk and participants' body, (2) "straight-ahead" gaze towards participants' eyes, (3) "down" - towards the table, (4) "left" - towards left screen, and (4) "right" - towards right screen (exact xyz coordinates (in $\mathrm{m}$ ) are provided in Table 1, measured from the robot frame of reference, i.e. waist).

iCub moved both its eyes and its neck to indicate the respective screen. The eyes and the neck of iCub were controlled by the YARP Gaze Interface, iKinGazeCtrl [30]. The vergence of the robot's eyes was set to 5 degrees and maintained constant. The trajectory time for the movement of eyes and neck was set to $200 \mathrm{~ms}$ and $400 \mathrm{~ms}$ respectively, to maintain the impression of a smooth and naturalistic movement. iCub's movements, triggers sent to the EEG recording system, presentation of stimuli, and data collection were controlled in OpenSesame (an open-source, graphical experiment builder for social sciences [31]) in combination with the iCub middleware YARP (Yet Another Robot Platform [32]), using the Ubuntu 12.04 LTS operating system.

TABLE I. Positions of robot gaze (measures in $\mathrm{m}$ )

\begin{tabular}{cccc}
\hline Positions of robot gaze & $\mathbf{x}$ & $\mathbf{y}$ & $\mathbf{z}$ \\
\hline Rest & -0.78 & 0.0 & 0.16 \\
Down & -0.78 & 0.0 & 0.04 \\
Left & -0.78 & -0.35 & 0.16 \\
Right & -0.78 & 0.35 & 0.16 \\
Straight-ahead & -0.78 & 0.0 & 0.28 \\
\hline
\end{tabular}

EEG was recorded with $\mathrm{Ag}-\mathrm{AgCl}$ electrodes from 64 electrodes of an active electrode system (ActiCap, Brain Products, $\mathrm{GmbH}$, Munich, Germany). Horizontal and vertical EOG were recorded bipolar from the outer canthi of the eyes and from above and below the observer's left eye, respectively. All electrodes were referenced to $\mathrm{AFz}$ and rereferenced offline to the average of all electrodes. Electrode impedances were kept below $10 \mathrm{k} \Omega$. Sampling rate was 500 $\mathrm{Hz}$, and the EEG activity was amplified with a band-pass filter of 0.1-250 Hz, BrainAmp amplifiers (Brain Products, GmbH).

\section{Procedure}

The whole session, including EEG preparation, lasted around 2.5 hours. Every trial consisted of the following steps: The trial started with the robot having its eyes closed, see Figure 1A. After 2s, the robot opened its eyes for $500 \mathrm{~ms}$ looking at the same position ("rest"), see Figure 1B. Following this delay, iCub looked either down ("down" gaze) or up towards the eyes of the participant ("straight-ahead" gaze), see Figure $1 \mathrm{C}$. The whole duration of this phase was $2 \mathrm{~s}$. The experiment was divided on 20 blocks of 16 trials, pseudorandomly assigned to the "straight-ahead" or "down" condition. Subsequently, the robot shifted to gaze to the left or right screen, see Figure 1D. After $500 \mathrm{~ms}$ delay from the initiation of this movement (Stimulus Onset Asynchrony, $\mathrm{SOA}=500 \mathrm{~ms}$ ), the letter $\mathrm{V}$ or $\mathrm{T}$ appeared on the same or the 
opposite screen (50\% probability) for $200 \mathrm{~ms}$, see Figure 1E. Participants were asked to respond as fast and as accurate as possible to the target identity by pressing the $\mathrm{V}$ button with their left hand for $\mathrm{V}$, and the $\mathrm{T}$ button with their right hand for $\mathrm{T}$ (Group 1), and the opposite stimulus-response mapping for Group 2. The screens remained blank (Figure 1F) until participant's response was executed, and the next trial started with robot closing its eyes at "rest" position. If participants did not respond within $1500 \mathrm{~ms}$, a new trial started, and the participant's response was registered as incorrect. At the end of every block, participants received feedback about their mean reaction time (RT) and accuracy. The order of the gaze blocks (straight-ahead or down) was counterbalanced between participants. The direction of the robot gaze, the identity of the letters and the screen of stimulus presentation were counterbalanced and randomly selected within each block. Participants had a practice session of two blocks (10 trials each) of both gaze conditions but with a random order. Participants had self-paced breaks after every block (1-2 mins), short breaks every 4 trials (3-5 mins) and a longer pause at the middle of the experiment ( 10 minutes $)$, in order to reduce fatigue.

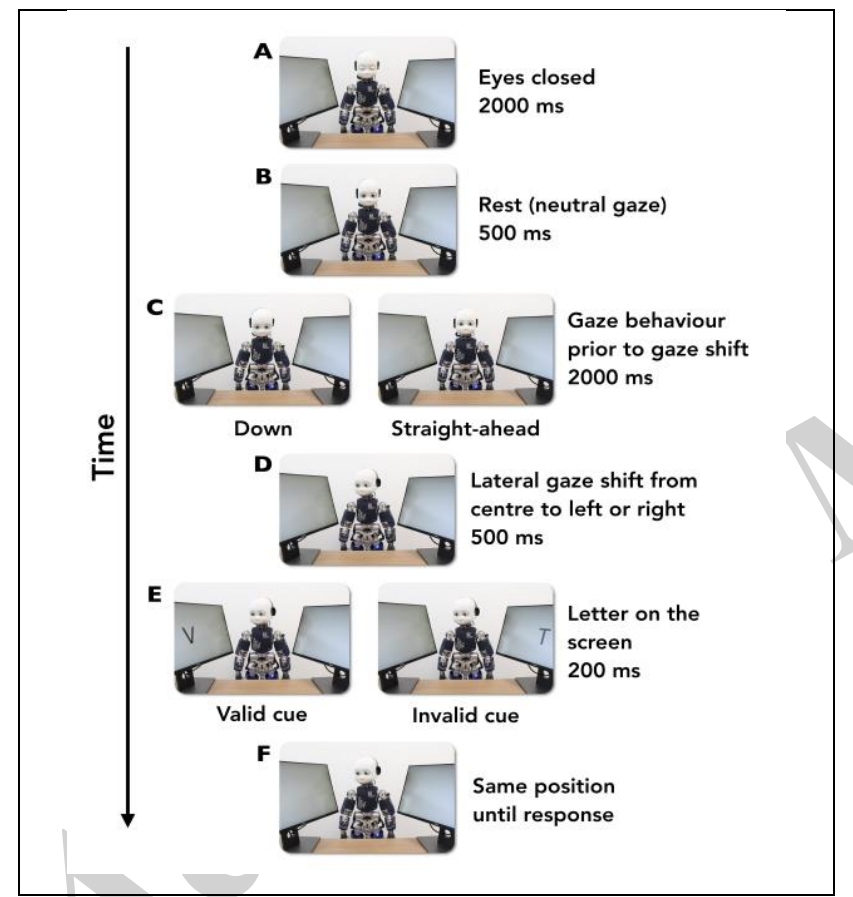

Figure 1. Experimental sequence.

C. Analysis

1) Behavioral data

For behavioral data, error trials $(4.02 \%)$, RTs $<100 \mathrm{~ms}$, or 2.5 SDs above- or below an individual's mean for each condition were removed $(2.53 \%$ of correct trials). We conducted analyses on the correct RTs of target discrimination. In order to determine whether there were any statistically significant differences between the means of the conditions, following standard statistical procedures, a repeated-measures analyses of variance (ANOVA) [33] were conducted on mean RTs, with gaze type (straight-ahead, down) and validity (valid, invalid) as within-subjects factors.
T-tests were conducted to compare RT means between valid and invalid conditions for the different gaze type conditions.

\section{2) EEG data pre-processing}

For the ERP analysis, we first filtered the raw data offline using a $30 \mathrm{~Hz}$ high-cutoff filter. Then we averaged the data over 1000-ms epochs including a $200 \mathrm{~ms}$ pre-stimulus baseline, time-locked to the target onset. For eye-movement artefacts, we inspected the F10, F9, and Fp1 channels using an automatic artefact-rejection procedure. We excluded trials with eye movements and blinks on either of these channels prior to averaging. Artefacts were defined as any absolute voltage difference in a segment exceeding $50 \mu \mathrm{V}$ or voltage steps between two sampling points exceeding $80 \mu \mathrm{V}$. We also excluded individual channels with other artefacts (all channels considered) if amplitude exceeded $\pm 80 \mu \mathrm{V}$ or activity was lower than $0.10 \mu \mathrm{V}$ for a $100 \mathrm{~ms}$ interval. The epochs were baseline-corrected with $200 \mathrm{~ms}$ period prior to stimulus onset.

\section{3) ERP analyses of the EEG signal}

To examine the ERP correlates of the behavioral gazecueing effect, we focused on the P1/N1 components, locked to the target onset. For the P1 and N1 mean amplitude analyses, we selected a time window based on the average latency of the grand-average peak for all conditions over the P3, P4, PO3 and PO4 channels (pooled). For P1, we selected the time window of $105-145 \mathrm{~ms}$ ( $\pm 20 \mathrm{~ms}$, relative to the peak latency), while for the N1 component we selected the time window of 150$230 \mathrm{~ms}$ ( $\pm 40 \mathrm{~ms}$ relative to the peak latency). For peak latency analyses on the P1/N1 components, we followed analogous procedure (and same time windows) as for the mean amplitude analyses. The mean amplitudes and peak latencies were subjected to separate $2 \times 2$ repeated-measures ANOVAs with gaze type (straight-ahead vs. down) and validity (valid vs. invalid) as within-subject factors. Planned comparisons ( $\mathrm{t}-$ tests) were conducted for the valid versus invalid conditions for the different gaze type conditions. Where appropriate, statistics were corrected according to Greenhouse-Geisser for potential nonsphericity.

The experiment had 80 repetitions per condition. After rejection of eye movement artefacts and incorrect-response trials, 71 trials (on average) remained in each experimental condition (straight-ahead valid: 70.8, invalid: 70.4; down valid: 72.3, invalid: 70.9). Letter ("V"/"T") and side of presentation (left/right) were averaged together.

\section{RESULTS}

\section{A. Behavioral}

The $2 \times 2$ ANOVA with the factors gaze type (straightahead vs. down) and validity (valid vs. invalid) on RTs revealed a significant main effect of validity, $F(1,20)=34.0$, $p<.001, \eta_{p}^{2}=.63$. This means that participants followed the gaze of the robot and were faster in valid (Mvalid $=438.05$, $\mathrm{SEM}=12.11$ ) relative to invalid trials (Minvalid $=452.86$, SEM $=10.88)$. There was no significant main effect of gaze type, $F(1,20)=2.85, p=.11, \eta_{p}^{2}=.12$, or interaction, $F(1$, $20)<1$. This indicates that there was no difference between straight and down gaze condition. However, we conducted pairwise comparisons to evaluate the validity effect within 
each gaze type condition. These analyses showed significant differences between valid and invalid trials, both in the straight-ahead gaze, $t(20)=4.51, p<.001(\mathrm{M} v$ alid $=435, \mathrm{SEM}$ $=10.41$; Minvalid $=450.5, \mathrm{SEM}=11.21)$ and the down-gaze condition, $t(20)=5.21, p<.001($ Mvalid $=441.1, \mathrm{SEM}=11.7$; Minvalid $=455.3, \mathrm{SEM}=13.2$ ), $\mathrm{cf}$. Figure 2. This means that participants followed the gaze of the robot in both gaze type conditions.

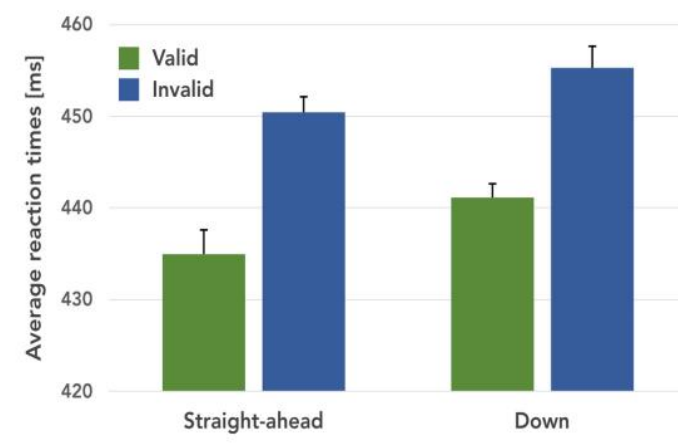

Figure 2. Average RTs. Left: straight-ahead gaze, right: down. Green bars: valid trials, blue bars: invalid trials. Error bars represent standard error of the means adjusted to within-participant designs according to Cousineau [34].

\section{B. EEG data}

\section{1) P1 component}

Analysis of mean amplitudes in the P1 time window between 105-145 ms post-target onset revealed no main effect of validity, $F(1,20)=1.14, p=.3, \eta_{p}^{2}=0.05$. There was no significant main effect of gaze type, $F(1,20)=1.8, p=$ $.19, \eta_{p}^{2}=0.08$, or interaction, $F(1,20)=3.89, p=.06, \eta_{p}^{2}=$ 0.16 . Also, peak latency analyses did not reveal any significant effect or interaction for P1 ( $p>.25)$, cf. Figure 3. Thus, results on the P1 component reveal that there was no attentional suppression observed for uncued locations.

\section{2) N1 component}

Mean amplitudes in the N1 time window (150-230 ms) post-target onset revealed a main effect of validity, $\mathrm{F}(1,20)=$ 5.21, $\mathrm{p}=0.034, \eta_{p}^{2}=0.21$ (Mvalid $=-1.21, \mathrm{SEM}=0.38$; Minvalid $=-0.92, \mathrm{SEM}=0.43)$. N1 is a typical EEG correlate of behavioural GCE, and suggests enhanced discriminative processing of stimuli within the focus of attention. There was no main effect of gaze type, $\mathrm{F}(1,20)=<1$, or interaction, $\mathrm{F}(1$, $20)<1$. Peak latency showed main effect of validity, $F(1,20)$ $=18.33, \mathrm{p}<.001, \eta_{p}^{2}=0.48$ and no interaction, $\mathrm{F}(1,20)<2.6$, cf. Figure 3, suggesting that the cued targets evoked not only enhanced processing at the attended location, but that the cued target was also processed faster.

\section{DISCUSSION}

The present study replicated, in an HRI setup the effect of gaze cueing at a neural and behavioural level. GCE is a phenomenon well established in cognitive neuroscience. This study is novel in showing that an embodied humanoid robot with mechanistic eyes and human-like face evokes similar attentional mechanisms as another human would do. The study also served as a proof-of-concept of integrating cognitive neuroscience methods, with an interactive HRI paradigm.

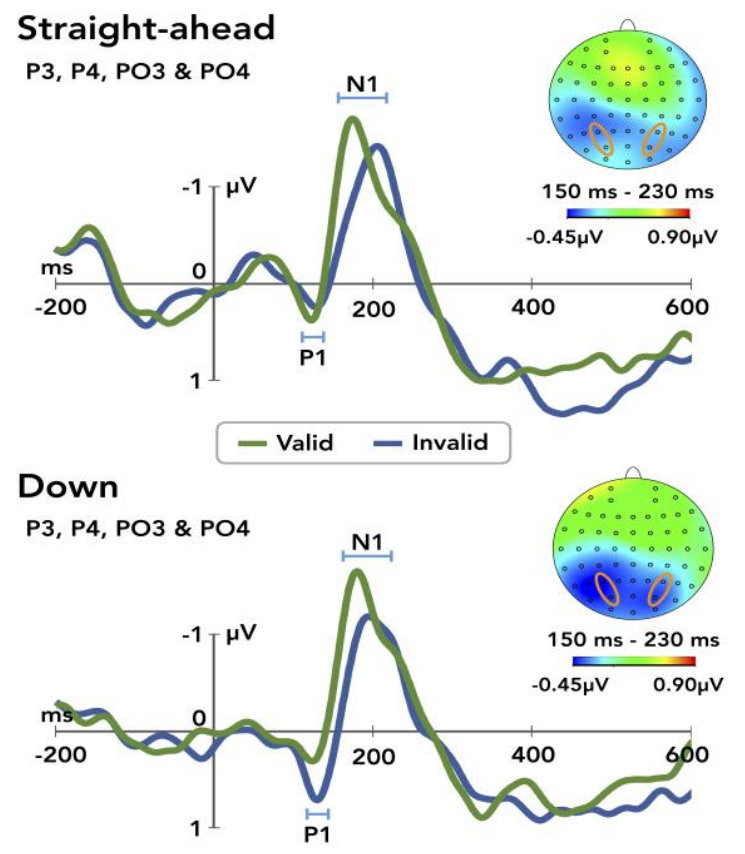

Figure 3. Grand-averaged ERP waveforms time-locked to target onset (left), and voltage distribution of the differential effect (right) for the straightahead gaze condition (top) and down condition (bottom). Green lines: validly cued trials, blue lines: invalidly cued trials. Time windows: P1, 115-135 ms; and N1, 150-230 ms. Red ovals mark the electrodes of interest (PO3/4, P3/4)

Furthermore, the results showed that the GCE induced by the robot generalize across different types of gaze conditions, namely gaze straight-ahead and gaze-down, prior to directional gaze cueing. It is important to note, however, that this might be the case only under the condition of 50\% validity and specific parameters of the experimental design (for example, the length of the SOA). Future research needs to examine all potential factors that might contribute to evoking the GCE in HRI.

In more detail, our results show that reaction times were faster, the N1 ERP component peaked earlier and had higher amplitude on validly cued trials, relative to invalidly cued trials. Faster reaction times in valid, compared to invalid, trials (GCE) indicate that participants engaged in JA with iCub, although the gaze was not predictive of target location. At the neural level, the amplitude and latency effects of the N1 component paralleled the behavioural results, and indicate that processing of stimuli at locations at which attention is focused due to gaze of the robot is enhanced [25]. Additionally, an earlier peak of $\mathrm{N} 1$ for valid vs. invalid trials indicates that stimuli at the attended trials are processed also faster, perhaps due to a lesser cognitive load [35].

In general, our findings suggest that participants followed the gaze of the robot in a reflexive and automatic manner, regardless lack of predictivity in the gaze and independent of the robot's gaze behavior prior to the directional shifts. This suggest that a humanoid robot $\mathrm{iCub}$ in a natural HRI scenario is capable of effectively orienting attention of observers towards the direction of its gaze, similarly to a human agent [10]. In the context of previous studies [13] which showed reduced GCE for robot faces on the screen, as compared to human faces, this provides a strong evidence that embodiment of a robotic agent plays a crucial role in engaging in JA [16]. 
In general, our study is a prime example of an approach linking neuroscience and robotics in order to establish properly scientifically-grounded HRI solutions. It can provide guidelines to robot designs on how the robot should behave in order to elicit well-defined and specific -but fundamental and crucial-brain mechanisms involved in social interactions. It is the very same mechanisms that are evoked in human-human social encounters. If robots are to co-exist with humans in the day-to-day social environment, they need to evoke those automatic and often implicit mechanisms of the human brain, in the exact same ways as other humans do. However, many present approaches in HRI research lack systematicity, and, with self-reported measures, do not tap on those fundamental (and often implicit) mechanisms of social cognition. To advance in HRI research we should first understand and measure (with well-controlled (neuro-)scientific or psychological methods) how humans respond to robots, then take these insights and translate them into improved robot design. In the present study we showed that it is feasible to (i) implement an experimental paradigm of cognitive neuroscience in an HRI protocol, and integrate all the necessary components, such as EEG, stimuli presentation, behavioural measures, (ii) obtain well documented effects from human-human interaction in HRI; (iii) observe fundamental mechanisms of social cognition being evoked by a humanoid robot. This is a promising avenue to design robots properly attuned to the workings of the human brain.

\section{REFERENCES}

C. Bartneck, D. Kulić, E. Croft, and S. Zoghbi, "Measurement Instruments for the Anthropomorphism, Animacy, Likeability, Perceived Intelligence, and Perceived Safety of Robots," Int. J. Soc. Robot., vol. 1, no. 1, pp. 71-81, Jan. 2009.

[2] C. L. Bethel and R. R. Murphy, "Review of Human Studies Methods in HRI and Recommendations," Int. J. Soc. Robot., vol. 2, no. 4, pp. 347-359, Dec. 2010.

[3] E. Wiese, G. Metta, and A. Wykowska, "Robots As Intentional Agents: Using Neuroscientific Methods to Make Robots Appear More Social," Front. Psychol., vol. 8, p. 1663, Oct. 2017.

[4] C. Moore, Joint attention: Its origins and role in development. Psychology Press, 2014.

[5] N. J. Emery, E. N. Lorincz, D. I. Perrett, M. W. Oram, and C. Baker, "Gaze following and joint attention in rhesus monkeys (Macaca mulatta).," J. Comp. Psychol., vol. 111, no. 3, pp. 286293, 1997.

[6] T. Grossmann and M. H. Johnson, "Selective prefrontal cortex responses to joint attention in early infancy," Biol. Lett., vol. 6, no. 4, pp. 540-543, 2010.

[7] M. Tomasello, Origins of human communication. MIT press, 2010.

[8] C. Friesen and A. Kingstone, "The eyes have it! Reflexive orienting is triggered by nonpredictive gaze," Psychon. Bull. Rev., 1998.

[9] A. Frischen, A. P. Bayliss, and S. P. Tipper, "Gaze cueing of attention: visual attention, social cognition, and individual differences.," Psychol. Bull., vol. 133, no. 4, p. 694, 2007.

[10] J. Driver, G. Davis, P. Ricciardelli, P. Kidd, E. Maxwell, and S. Baron-Cohen, "Gaze perceptiontriggers reflexive visuospatial orienting," Vis. cogn., vol. 6, no. 5, pp. 509-540, 1999.

[11] H. Admoni, C. Bank, J. Tan, M. Toneva, and B. Scassellati, "Robot gaze does not reflexively cue human attention," Proc. Annu. Meet. Cogn. Sci. Soc., vol. 33, no. 33, pp. 1983-1988, 2011.

[12] Y. Okumura, Y. Kanakogi, T. Kanda, H. Ishiguro, and S. Itakura, "Infants understand the referential nature of human gaze but not robot gaze.," J. Exp. Child Psychol., vol. 116, no. 1, pp. 86-95, Sep. 2013.

[13] E. Wiese, A. Wykowska, J. Zwickel, and H. J. Müller, "I See What You Mean: How Attentional Selection Is Shaped by Ascribing Intentions to Others," PLoS One, vol. 7, no. 9, p. e45391, Sep. 2012.
[14] G. Metta, L. Natale, F. Nori, G. Sandini, D. Vernon, L. Fadiga, C. von Hofsten, K. Rosander, M. Lopes, J. Santos-Victor, A. Bernardino, L. Montesano L, "The iCub humanoid robot: An opensystems platform for research in cognitive development". Neural Networks, 23(8-9): 1125-1134.

[15] A. Wykowska, J. Kajopoulos, and K. Ramirez-Amaro, "Autistic traits and sensitivity to human-like features of robot behavior," Interact. Stud., vol. 16, no. 2, pp. 219-248, 2015.

[16] A. Wykowska, T. Chaminade, and G. Cheng, "Embodied artificial agents for understanding human social cognition," Phil. Trans. R. Soc, vol. 371, no. 1693, p. 20150375, 2016.

[17] H. Admoni and B. Scassellati, "Social Eye Gaze in Human-Robot Interaction: A Review," J. Human-Robot Interact., vol. 6, no. 1, pp. 25-63, 2017.

[18] A. Sciutti, C. Ansuini, C. Becchio, and G. Sandini, "Investigating the ability to read others' intentions using humanoid robots," Front. Psychol., vol. 6, 2015.

[19] A. M. Schuller and B. Rossion, "Spatial attention triggered by eye gaze increases and speeds up early visual activity.," Neuroreport, vol. 12, no. 11, pp. 2381-6, Aug. 2001.

[20] G. R. Mangun and S. A. Hillyard, "Allocation of visual attention to spatial locations: tradeoff functions for event-related brain potentials and detection performance.," Percept. Psychophys., vol. 47, no. 6, pp. 532-50, Jun. 1990.

[21] S. J. Luck, G. F. Woodman, and E. K. Vogel, "Event-related potential studies of attention," Trends Cogn. Sci., vol. 4, no. 11, pp. 432-440, Nov. 2000.

[22] H. L. Hawkins, S. A. Hillyard, S. J. Luck, M. Mouloua, C. J. Downing, and D. P. Woodward, "Visual attention modulates signal detectability.," J. Exp. Psychol. Hum. Percept. Perform., vol. 16, no. 4, pp. 802-11, Nov. 1990.

[23] H. J. Müller and J. M. Findlay, "The effect of visual attention of peripheral discrimination thresholds in single and multiple element displays," Acta Psychol. (Amst)., vol. 69, no. 2, pp. 129-155, Nov. 1988.

[24] G. Mangun, S. Hillyard, and S. Luck, "IQ electrocortical substrates of visual selective attention," Atten. Perform. XIV Synerg. Exp. Psychol. Artif. Intell. Cogn. Neurosci., vol. 14, p. 219, 1993.

[25] S. A. Hillyard, E. K. Vogel, and S. J. Luck, "Sensory gain control (amplification) as a mechanism of selective attention: electrophysiological and neuroimaging evidence.," Philos. Trans. R. Soc. Lond. B. Biol. Sci., vol. 353, no. 1373, pp. 1257-70, Aug. 1998.

[26] J. R. Fedota, C. G. McDonald, D. M. Roberts, and R. Parasuraman, "Contextual task difficulty modulates stimulus discrimination: Electrophysiological evidence for interaction between sensory and executive processes," Psychophysiology, vol. 49, no. 10, pp. 13841393, Oct. 2012.

[27] A. Wykowska, E. Wiese, A. Prosser, and H. J. Müller, "Beliefs about the minds of others influence how we process sensory information," PLoS One, vol. 9, no. 4, 2014.

[28] J. Perez-Osorio, H. J. Müller, E. Wiese, and A. Wykowska, "Gaze following is modulated by expectations regarding others' action goals," PLoS One, vol. 10, no. 11, 2015.

[29] F. Faul, E. Erdfelder, A. Lang, and A. Buchner, "G* Power 3: A flexible statistical power analysis program for the social, behavioral, and biomedical sciences," Behav. Res. Methods, vol. 39, no. 2, pp. 175-191, 2007.

[30] A. Roncone, U. Pattacini, G. Metta, and L. Natale, "A Cartesian 6DoF Gaze Controller for Humanoid Robots.," in Proceedings of Robotics: Science and Systems, 2016.

[31] S. Mathôt, D. Schreij, and J. Theeuwes, "OpenSesame: An opensource, graphical experiment builder for the social sciences," Behav. Res. Methods, vol. 44, no. 2, pp. 314-324, Jun. 2012.

[32] G. Metta, P. Fitzpatrick, and L. Natale, "YARP: Yet Another Robot Platform," Int. J. Adv. Robot. Syst., vol. 3, no. 1, p. 8, Mar. 2006.

[33] A. Field, "Discovering Statistics Using IBM SPSS Statistics", SAGE Publications, 2013.

[34] D. Cousineau, "Confidence intervals in within-subject designs: A simpler solution to Loftus and Masson's method," Tutorials in quantitative methods for psychology, 1(1), 42-45, 2005.

[35] E. Callaway and R. Halliday, "The effect of attentional effort on visual evoked potential N1 latency," Psychiatry Res., vol. 7, no. 3, pp. 299-308, Dec. 1982. 